\title{
STATE DEPENDENT VOLUME ISOTOPE SHIFT ANALYSIS OF THE LOW LYING STATES OF Ba I and Ba II.
}

\author{
B. FRICKE ${ }^{1}$, P. GRUNDEVIK, I. LINDGREN, G. OLSSON, \\ T. OLSSON, A. ROSÉN and G. TORBOHM ${ }^{1}$ \\ Department of Physics Chalmers University of Technology, S-412 96 Göteborg, Sweden
}

Received 25 May 1983

\begin{abstract}
Relativistic multi-configuration Dirac-Fock wavefunctions, coupled to good angular momentum $J$, have been calculated for low lying states of $\mathrm{Ba} \mathrm{l}$ and $\mathrm{Ba}$ II. The resulting electronic factors show good agreement with data derived from recent high-resolution laser spectroscopy experiments and results from a comparison of muonic and optical data.
\end{abstract}

The electron density at the nucleus is a very important quantity in a number of measurements (isomershifts, optical-, X-ray, and muonic-isotope shifts), where electronic and nuclear operators describe the interaction [1]. In order to extract the nuclear charge distribution or the difference of this quantity between different isotopes from this kind of measurements, the knowledge of the electronic density at the nucleus is absolutely necessary. For a long time several groups have tried to extract nuclear charge distribution parameters from consistent exploration of muonic and optical spectroscopy data using ab-initio or semi-empirical estimations of the electron density at the nucleus. This is in principle possible as both experiments measure different in tegral quantities caused by differences of the nuclear and electronic charge densities.

In a theoretical treatment of the isotope shift (IS) it is important to include the effect of relativity and configuration interaction. In nearly all publications each of these effects have been analyzed separately, only. Ref. [2] is a good example of the discussion of relativity whereas ref. [3] is an extended but non-relativistic discussion of the influence of configuration interaction in the isotope-shift. Grant [4] has published an exploratory work of the influence of both effects on the volume isotope-shift. We present here the results

1 Permanent address: Department of Physics, University of Kassel, Germany. of ab-initio relativistic state-dependent multi-configuration Dirac-Fock (MCDF) calculations for the charge density at the nucleus for low lying states of Ba I and $\mathrm{Ba}$ II. The calculations are performed with the MCDFcomputer program of Desclaux [5]. In this type of calculations relativity as well as certain types of configuration mixing between open shell electrons are included directly in the selfconsistent procedure. This is absolutely necessary since both effects are large and affect each other.

The isotopic shift in an atomic transition $i$ is given as (neglecting higher order tems in the nuclear charge distribution) $[1,2]$ :

$\delta_{\nu_{i}}^{\mathrm{AA}^{\prime}}=F_{i} \delta\left\langle r^{2}\right\rangle^{\mathrm{AA}^{\prime}}+M_{i}\left(m_{\mathrm{A}^{\prime}}-m_{\mathrm{A}}\right) / m_{\mathrm{A}} m_{\mathrm{A}^{\prime}}$, where the first term represents the volume shift and the second term the mass shift. $F_{i}$ is the electronic factor of the volume shift and $\delta\left\langle r^{2}\right\rangle^{\mathrm{AA}^{\prime}}$ the difference of nuclear mean-square charge radii. In this work we will calculate the volume-isotope shift, only.

Since the electronic charge density varies strongly over the nuclear volume we use an extended nucleus with a two-parameter Fermi-nuclear charge distribution [6] and 21 mesh-points inside the nuclear radius. The electronic charge densities presented in this paper are always values at the center of the nucleus. The five lowest levels with positive parity and the 16 lowest levels with negative parity, all originating from the atomic levels $6 \mathrm{~s}_{1 / 2}, 6 \mathrm{p}_{1 / 2}, 6 \mathrm{p}_{3 / 2}, 5 \mathrm{~d}_{3 / 2}$, and $5 \mathrm{~d}_{5 / 2}$, 


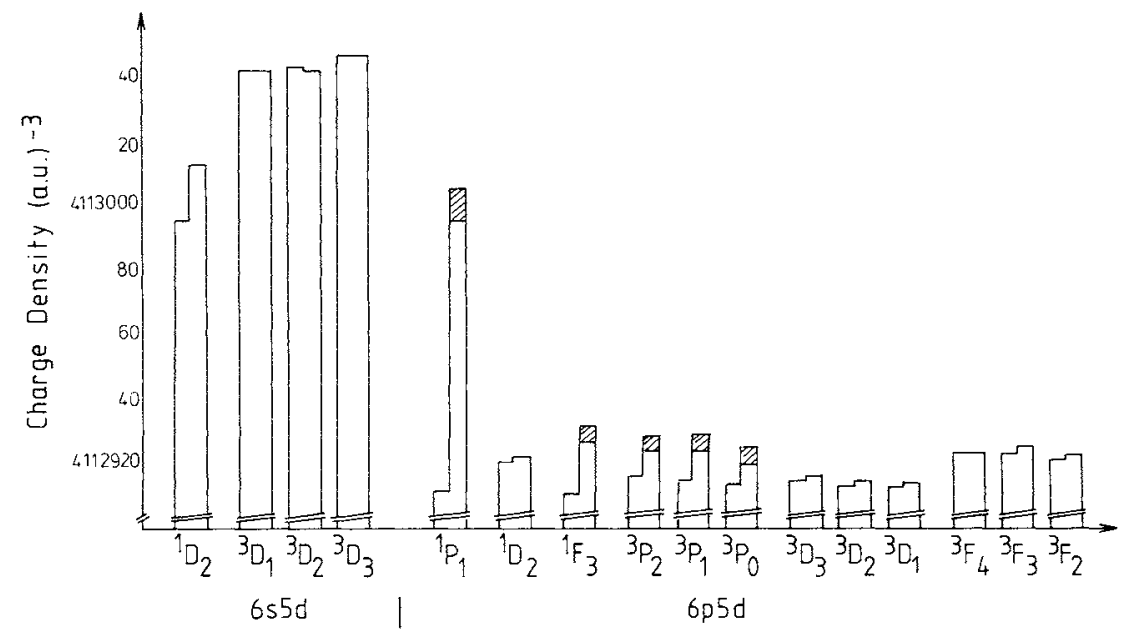

Fig. 1. Electron densities at the nucleus for $\mathrm{Ba} I$ in the configuration $6 \mathrm{~s} 5 \mathrm{~d}$ and $6 \mathrm{p} 5 \mathrm{~d}$ calculated with an MCDF program. For each state two different values are shown in the columns. The values to the left have been calculated for each state by mixing within the $6 \mathrm{~s} 5 \mathrm{~d}$ and $6 \mathrm{p} 5 \mathrm{~d}$ configurations, respectively. The values to the right are obtained by mixing of the configurations resulting from the wavefunctions $6 \mathrm{~s}, 6 \mathrm{p}, 5 \mathrm{~d}$. The shaded area shows the uncertainty due to convergence problems.

coupled to good angular momentum $J$, were calculated. In addition, calculations were performed for the $6 s_{1 / 2}$, $6 \mathrm{p}_{1 / 2}$, and $6 \mathrm{p}_{3 / 2}$ states of Ba II. These results are compared with experimental volume shifts of each combination of optical transitions in the low lying spectrum of $\mathrm{Ba}$ I derived from recent high-resolution laser spectroscopy measurements [2,7]. In addition electronic factors are also available from muonic data [8] which are used for a further check of the calculations.

The electronic charge density $4 \pi \rho$ at the nucleus for the lowest states of positive parity ( $6 \mathrm{~s} 5 \mathrm{~d}$ configuration) is given in fig. 1 . For each state the value $a b$ tained for coupling within the main configuration is given to the left of each column. To the right in each column in fig. 1 we give the electronic charge density obtained by mixing of different configurations with the same parity, i.e. $\left(6 s^{2}, 6 s 5 d, 6 p^{2}, 5 d^{2}\right)$ and $(6 s 6 p$, $6 \mathrm{p} 5 \mathrm{~d})$. There is a significant change by including full mixing (i.e. taking in to account all possible states resulting from the levels discussed here), particularly for the singlet states. For example the ${ }^{1} \mathbf{P}_{1}$ states designated as $6 \mathrm{~s} 6 \mathrm{p}$ and $6 \mathrm{p} 5 \mathrm{~d}$ changed by -75 and +90 (in units used in fig. 1) when the admixtures of these configurations were taken into account.

In the analysis of isotope shifts a King plot procedure is normally performed. The isotope shifts in one transition $i$ are plotted as a function of the correspond- ing isotope shifts in another transition $j$, known as the reference. The slope of the resulting line is given as the ratio between the changes in electron density at the nucleus $\Delta \rho_{i} / \Delta \rho_{j}$ or in terms of the factors $F_{i} / F_{j}$ for the two transitions $i$ and $j$. Following this type of analysis of the high-resolution laser spectroscopy measurements between the $6 \mathrm{~s} 5 \mathrm{~d}$ and $6 \mathrm{p} 5 \mathrm{~d}$ configurations

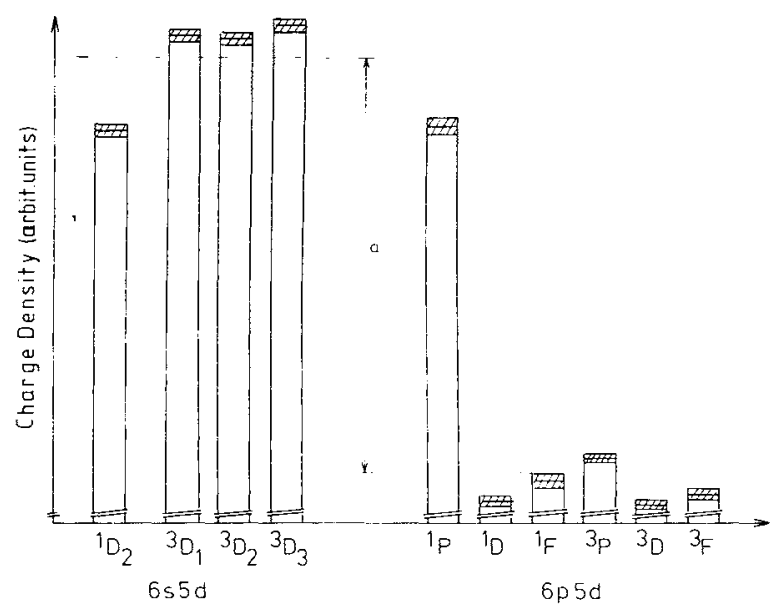

Fig. 2. Experimental electron densities at the nucleus as evaluated from experimental high-resolution laser spectroscopy measurements. The shaded area shows the experimental uncertainty. 
Table 1

Comparison of experimental and theoretical slopes of lines in a King plot analysis with the $6 s^{2}{ }^{1} \mathrm{~S}_{0}-6 \mathrm{~s} 6 \mathrm{p}{ }^{3} \mathrm{P}_{1}$ transition at 7911 $A$ as the reference.

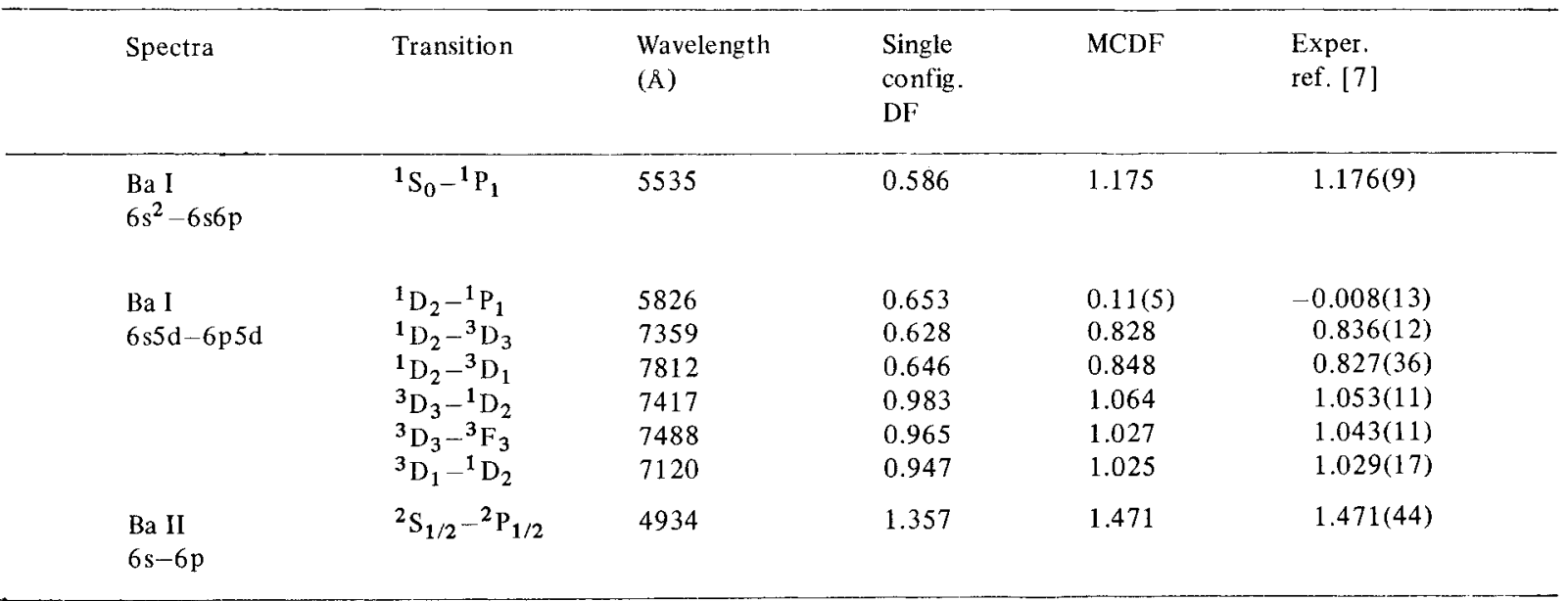

the relative difference in the charge density at the nucleus for the states in the two configurations was derived [7]. The result is shown in fig. 2. The distance " $a$ " in the figure corresponds to the average change in the electron density $\Delta \rho(6 s 5 \mathrm{~d}-6 \mathrm{p} 5 \mathrm{~d})$ given in arbitrary units. The deviation from the average for each state is clearly observed. We notice how the charge densities, evaluated from the MCDF wavefunctions displayed in fig. 1, resemble the experimental structure in fig. 2 . We also observe the significant improvement achieved when the full MCDF wavefunctions are used compared with DF-calculations within one configuration (fig. 1). The comparison between the theoretical and experimental results are explicitly given in table 1 . The values are presented as ratios $F_{i} / F_{j}$ (i.e. slopes in a King plot analysis) of different transitions, with the $6 \mathrm{~s}^{2}{ }^{1} \mathrm{~S}_{0}$ $-6 s 6 p{ }^{3} P_{1}$ transition as the reference. The experimental values are fully reproduced within the error by the MCDF-calculations. This is even true for transitions to the strongly disturbed ${ }^{1} \mathrm{P}_{1}$ states.

However, to be able to extract the change in the mean-square radius $\delta\left\langle r^{2}\right\rangle$ from the measured isotope shifts, known values of the specific mass shift and the electronic factor $F_{i}$, not the ratio $F_{i} / F_{j}$, of the volume shifts are required. In table $2 F_{i}$ values for some transsitions, calculated with different ab-initio methods, are given.

The values in the fourth column have been calculated

\section{Table 2}

Comparison of different theoretical ab initio calculations of the changes in electron density at the nucleus for some transitions.

\begin{tabular}{|c|c|c|c|c|c|c|}
\hline \multirow[t]{2}{*}{ Spectra } & \multirow[t]{2}{*}{ Transition } & \multirow{2}{*}{$\begin{array}{l}\text { Wavelength } \\
(\AA)\end{array}$} & \multicolumn{4}{|c|}{$F_{i}\left(\mathrm{GHz} / \mathrm{fm}^{2}\right)$} \\
\hline & & & $\begin{array}{l}\text { Average } \\
\text { DF } \\
\text { ref. [7] }\end{array}$ & $\begin{array}{l}\text { Single } \\
\text { config. } \\
\text { DF }\end{array}$ & $\mathrm{MCDF}$ & $\begin{array}{l}\text { Exper. } \\
\text { ref. }[8]\end{array}$ \\
\hline $\begin{array}{l}\text { Ba I } \\
6 s^{2}-6 s 6 p\end{array}$ & $\begin{array}{l}{ }^{1} S_{0}-{ }^{3} P_{1} \\
{ }^{1} S_{0}-{ }^{1} P_{1}\end{array}$ & $\begin{array}{l}7911 \\
5535\end{array}$ & -2.34 & $\begin{array}{l}-2.67 \\
-1.56\end{array}$ & $\begin{array}{l}-2.55 \\
-2.99\end{array}$ & $\begin{array}{l}-2.59(22) a \\
-3.04(26)\end{array}$ \\
\hline
\end{tabular}

a) These values have been calculated from the value for the $5535 \AA$ transition $[3.04(26)]$ using the experimental slopes in table 1 . 
from Dirac-Fock (DF) wavefunctions [7] evaluated for the average energy of the terms achieved in the particular configuration [9]. These values have been evaluated assuming a uniform charge distribution with the nuclear radius $R_{\mathrm{A}}=1.2 A^{1 / 3} \mathrm{fm}$. The single DF and MCDF values presented in column 5 and 6 have been calculated with a nuclear Fermi charge distribution. The values are given as the electronic factor corrected for the change of the electronic charge density over the nuclear volume. In connection with recent muonic X-ray measurements a King plot analysis was performed [8]. In a plot of the optical isotope shift in the $5535 \AA$ transition versus the muonic $\delta\left\langle r^{2}\right\rangle$ values the electronic factor $F_{5535}$ was estimated to be in good agreement with our result evaluated with the MCDF wavefunctions (table 2). The experimentally derived electronic factors for the $4934 \AA$ and $7911 \AA$ transitions - using $F_{5535}$ and the experimental slopes in table 1 - are also included in table 2. Some years ago Fischer et al. [10] estimated the factor $F_{4934}$ $=-4.98 \mathrm{GHz} / \mathrm{fm}^{2}$ by use of magnetic hyperfine structure data. It should, however, be noted that the mag. netic hyperfine operator and the fieldshift operator are relativistically quite different [11] and it is necessary to evaluate the electronic factor in the correct way. In addition, polarization and correlation effects affect the two operators differently. In the evaluation of nuclear mean-square radii from measured isotope shifts in the $6 s^{2}{ }^{1} S_{0} \rightarrow 6 s 6 p^{1} P_{1}$ transition the semiempirical value by Fischer et al. [10] has been used as the reference in the calculation of the electronic factor $[12,13]$. Just recently another value $F_{5535}$ $=-3.99(65) \mathrm{GHz} / \mathrm{fm}^{2}$ of the electronic factor in the $5535 \AA$ transition has been achieved from muonic data [14]. This value, with a rather big uncertainty, is in good agreement with the old semi-empirical value of Fischer et al. [10]. More consistent results of electronic factors derived from muonic experiments are therefore necessary for a further check.

From the comparison performed in this work it is evident that ratios of the change in electron density at the nucleus between different transitions as well as the absolute magnitude of the density can be well de- scribed by state dependent MCDF calculations. For this type of calculation it is not sufficient to use quantities evaluated for the average configuration and by including only the state dependency within one configuration but also the mixing of different configurations. The MCDF approach seems to work well for the low lying states, while calculations for higher lying states will probably be more difficult due to a strong mixing of many states. A more extensive analysis of the volume isotope shifts as well as the fine structure, the magnetic dipole- and electric quadrupole-in teraction will be given in a forthcoming paper.

This work was financially supported by the Swedish Natural Science Research Council (NFR). The authors thank L.M. Simon for information on new muonic data prior to publication. One of us (B.F.) acknowledges NORDITA and NFR for support during the visit in Sweden.

\section{References}

[1] J. Bauche and R.-J. Champeau, Adv. At. Mol. Phys. 12 (1976) 39.

[2] P. Grundevik, H. Lundberg, L. Nilsson and G. Olsson, Z. Phys. A306 (1982) 195.

[3] P. Aufmuth, J. Phys. B15 (1982) 3127.

[4] I.P. Grant, Phys. Scr. 21 (1980) 443.

[5] J.P. Desclaux, Comput. Phys. Commun. 9 (1975) 31.

[6] K.H. Hellwege, ed., Landolt-Börnstein, Zahlenwerte und Funktionen, Bd. 2, (Springer, Berlin, 1967).

[7] P. Grundevik, M. Gustavsson, G. Olsson and T. Olsson, to be published.

[8] E.B. Shera, H.D. Wohlfart and M.V. Hoehn, Phys. Lett. 112B (1982) 124.

[9] I. Lindgren and A. Rosén, Case studies, At. Phys. 4 (1974) 250.

[10] W. Fischer, M. Hartmann, H. Hühnermann and H. Vozz, Z. Phys. 267 (1974) 209.

[11] J. Bauche, Comm. Atom Mol. Phys. 10 (1981) 57.

[12] K. Bekk et al., Z. Phys. A291 (1979) 219.

[13] A.C. Mueller, F. Buchingern, W. Klempt, E.W. Otten, R. Neugart, C. Eckström and J. Heinemeier, Nucl. Phys., to be published.

[14] L.M. Simon, private communication. 